\title{
Prevalence of Helicobacter pylori infection and its association with lipid profiles
}

\author{
Sun $\mathrm{Y}^{1}$, Fu D ${ }^{2}$, Wang $\mathrm{YK}^{1}$, Liu $\mathrm{M}^{1}$, Liu $\mathrm{XD}^{1}$ \\ Department of Nuclear Medicine, Huashan Hospital, Fudan University, Shanghai, China. \\ xingdliu@yahoo.com
}

\begin{abstract}
OBJECTIVE: To evaluate the prevalence of Helicobacter pylori $(H$. pylori, HP) infection in subjects receiving routine physical examination and its associations with age, sex, body mass index (BMI) and lipid profiles. MATERIALS AND METHODS: Clinical information of 22,103 individuals who took routine physical examinations, including that on age, gender, height, weight, triglyceride, total cholesterol, high-density lipoprotein (HDL) cholesterol, low-density lipoprotein (LDL) cholesterol and data of HP infection were collected and analyzed. RESULTS: $H$. pylori infection rate in 22,103 subjects taking routine physical examination was $44.5 \%$. More men tended to be infected with $H$. pylori than women $(45.9 \%$ vs $42.8 \% ; p<0.01)$. The highest positive rate group was in the age group of 30-39 years (46.8\%) and the lowest rate was in the age group younger than 30 years $(40.5 \%)$. The obese had higher infection rate than the non-obese $(p<0.01)$. Mann-Whitney $U$ test was used to explore the relationships between lipid profiles and $H$. pylori infection. There were significant associations among HDL, triglyceride and HP infection $(p<0.01)$. However, significant differences were not confirmed between cholesterol, LDL and $H$. pylori infection.

CONCLUSION: H. pylori infection was common among subjects receiving physical examination in Shanghai and it was most significantly associated with HDL and triglyceride, indicating that $H$. pylori might be a new cardiovascular risk factor (Tab. 3, Ref. 23). Text in PDF www.elis.sk.

KEY WORDS: Helicobacter pylori, prevalence, triglyceride, cholesterol, HDL, LDL.
\end{abstract}

\section{Introduction}

H. pylori is regarded as a causative agent of digestive disorders such as chronic gastritis, peptic ulcer, gastric malignancies, and particularly gastric cancer (GC) and gastric mucosa-associated lymphoid tissue (MALT) lymphoma (1). It is classified as class I carcinogen by the International Agency for Research on Cancer. In the world, about one half or more people are infected with Helicobacter pylori (2). Moreover, most people infected with $H$. pylori are asymptomatic throughout their life (3-5). Although most subjects who received physical examination have no clinical symptoms of digestive disorders, they may be infected with H. pylori. Therefore it is important to investigate whether physical examination enhances infection with $H$. pylori or not.

Researchers reported that the prevalence of infection with $H$. pylori in obese subjects tends to be higher compared with the non-

${ }^{1}$ Department of Nuclear Medicine, Huashan Hospital, Fudan University, Shanghai, China, and ${ }^{2}$ Department of Nuclear Medicine, Shanghai 10th People's Hospital, Tongji University School of Medicine, Shanghai, China

Address for correspondence: Xingdang Liu, PhD, Department of Nuclear Medicine, Huashan Hospital of Fudan University, Wulumuqi M. Road 12, Shanghai 200040, China.

Phone: +86.215.2888308, Fax: +86.213 .2140391$

Acknowledgements: We express our gratitude to all people who participated in this study. We would like to thank the Medical Examination Center of Huashan Hospital Affiliated to Fudan University in Shanghai for providing us with technical help. obese $(6,7)$. But there were also some studies that showed no significant associations between BMI and $H$. pylori infection $(8,9)$. Thus the information regarding weight and height of patients was also collected to evaluate whether BMI was associated with HP infection.

A number of studies have demonstrated that $H$. pylori infection was significantly related to triglyceride, total cholesterol, LDL cholesterol and HDL cholesterol (9-11). But the results are not consistent between different studies. LDL cholesterol was significantly elevated in the subjects with $H$. pylori infection compared to those without $H$. pylori infection. There were no associations among other lipid profiles and $H$. pylori infection (9). However, another study showed that an $H$. pylori-positive group had lower HDL cholesterol than the H. pylori-negative group. Significant differences between the two groups were not found in other lipid profiles including triglyceride, total cholesterol and LDL cholesterol (12). So, the association between HP infection and lipid profiles still remains controversial.

Therefore, the aim of our study was to investigate the prevalence of $H$. pylori infection in subjects receiving physical examination and to find out whether there were significant associations between BMI, lipid profiles and $H$. pylori infection.

\section{Materials and methods}

\section{Subjects}

Retrospective study was conducted using the data of subjects who took physical examinations at the health examination center 
in Huashan Hospital Affiliated to Fudan University in Shanghai, China. We collected information on all individuals who were suspected for $H$. pylori infection from July 2013 to December 2014. A total of 22,103 individuals were included in this study. People who received medication therapy such as PPI or antibiotics during the past month were excluded.

This study was approved by the ethic committee of Huashan Hospital Affiliated to Fudan University.

\section{Information of the subjects}

The basic and laboratory information including sex, age, height, weight and values for triglyceride, total cholesterol, LDL cholesterol and HDL cholesterol were collected. Body mass index (BMI) was calculated as weight (kilograms) / height (meters) squared. According to BMI, three groups were formed: underweight $\left(\mathrm{BMI}<18.5 \mathrm{~kg} / \mathrm{m}^{2}\right)$, normal $\left(18.5 \leq \mathrm{BMI}<25 \mathrm{~kg} / \mathrm{m}^{2}\right)$ and overweight (BMI $\geq 25 \mathrm{~kg} / \mathrm{m}^{2}$ ). The age (14-99 years) was separated into five groups: $<30,30-39,40-49,50-59$ and $\geq 60$ years.

\section{Detection method}

H. pylori infection status was tested by ${ }^{13} \mathrm{C}$ urea breath test $\left({ }^{13} \mathrm{C}-\mathrm{UBT}\right)$. So far ${ }^{13} \mathrm{C}$-UBT with high specificity and high sensitivity has been regarded as the most reliable non-invasive method of detecting H. pylori (Maity et al, 2014). The kit for ${ }^{13} \mathrm{C}$-urea breath test including $75 \mathrm{mg}{ }^{13} \mathrm{C}$-urea powder was provided by Isodiagnostika Corporation (Canada). And the test machine was the AP2003 Mass Spectrometer. The critical value of the delta over baseline (DOB) of the ${ }^{13} \mathrm{CO}_{2} /{ }^{12} \mathrm{CO}_{2}$ ratio was 3.5. When the DOB was equal or higher than 3.5 , it was considered positive.

\section{Statistical analysis}

The data was presented as mean \pm standard deviation or percentages. The Chi-square test was used to see the percentages of $H$. pylori infection according to subgroups of age, sex and BMI. The significant differences in mean values for lipid profiles between $H$. pylori-positive and negative subjects were calculated by MannWhitney U test. All statistical analyses were performed using the Statistical Package for Social Science (version 18.0). The required significance level for the tests was chosen at the $\mathrm{p}$ value $<0.05$.

\section{Results}

\section{Characteristics of the subjects}

As described in Table1, 22,103 subjects were included in this study. Of them 11,582 (52.4\%) subjects were male and 10,521 (47.6\%) subjects were female. The mean values for age and BMI were $46.1 \pm 12.8$ years and $23.8 \pm 3.4 \mathrm{~kg} / \mathrm{m}^{2}$ respectively. The total values for triglyceride, total cholesterol, LDL cholesterol and HDL cholesterol were $1.65 \pm 1.39 \mathrm{mmol} / \mathrm{L}, 5.07 \pm 0.98 \mathrm{mmol} / \mathrm{L}$, $1.33 \pm 0.36 \mathrm{mmol} / \mathrm{L}$ and $2.97 \pm 0.79 \mathrm{mmol} / \mathrm{L}$.

Correlations between age, sex, BMI and H. pylori infection

Age was divided into five subgroups and BMI was divided into three subgroups as showed in Table 2 . The total prevalence of the $H$. pylori infection was $44.5 \%$. Significant differences were iden-
Tab. 1. Characteristics of the subjects.

\begin{tabular}{lccc}
\hline & $\begin{array}{c}\text { Total } \\
\mathrm{n}=22,103\end{array}$ & $\begin{array}{c}\text { Male } \\
\mathrm{n}=11,582\end{array}$ & $\begin{array}{c}\text { Female } \\
\mathrm{n}=10,521\end{array}$ \\
\hline Age $($ years $)$ & $46.1 \pm 12.8$ & $46.8 \pm 12.8$ & $45.4 \pm 12.7$ \\
BMI $\left(\mathrm{kg} / \mathrm{m}^{2}\right)$ & $23.8 \pm 3.4$ & $24.9 \pm 3.2$ & $22.6 \pm 3.2$ \\
Triglyceride $(\mathrm{mmol} / \mathrm{L})$ & $1.65 \pm 1.39$ & $2.00 \pm 1.64$ & $1.27 \pm 0.91$ \\
Cholesterol $(\mathrm{mmol} / \mathrm{L})$ & $5.07 \pm 0.98$ & $5.08 \pm 0.96$ & $5.06 \pm 1.00$ \\
HDL cholesterol $(\mathrm{mmol} / \mathrm{L})$ & $1.33 \pm 0.36$ & $1.19 \pm 0.26$ & $1.48 \pm 0.38$ \\
LDL cholesterol $(\mathrm{mmol} / \mathrm{L})$ & $2.98 \pm 1.71$ & $3.04 \pm 0.78$ & $2.90 \pm 0.80$ \\
\hline
\end{tabular}

BMI - body mass index; HDL cholesterol - high-density lipoprotein cholesterol; LDL cholesterol -low-density lipoprotein cholesterol. Values are mean \pm standard error of the mean

Tab. 2. Prevalence of $H$. pylori infection in different sex, age and BMI groups.

\begin{tabular}{lcccc}
\hline & & $\begin{array}{c}\text { H. pylori-positive } \\
\text { rate (\%) }\end{array}$ & $\begin{array}{c}\text { H. pylori-negative } \\
\text { rate (\%) }\end{array}$ & $\mathrm{p}$ \\
\hline Sex & Male & 45.9 & 54.1 & $<0.01$ \\
\hline & Female & 42.8 & 57.2 & \\
Age groups & $30-39$ & 40.5 & 59.5 & \\
(years) & $40-49$ & 46.8 & 53.2 & \\
& $50-59$ & 45.3 & 54.7 & $<0.01$ \\
& $\geq 60$ & 44.8 & 55.2 & \\
\hline \multirow{2}{*}{ BMI groups } & $<18.5$ & 42.6 & 58.4 & \\
$\left(\mathrm{~kg} / \mathrm{m}^{2}\right)$ & $18.5-24.9$ & 42.7 & 57.4 & \\
& $\geq 25$ & 47.9 & 57.3 & $<0.01$ \\
\hline Total & & 44.5 & 52.1 & \\
\hline BMI & & 55.5 & \\
\hline
\end{tabular}

BMI - body mass index; $p$ values by Chi-square test.

tified in respect of sex, age and BMI between $H$. pylori-positive group and $H$. pylori-negative group $(\mathrm{p}<0.01)$. Male had higher prevalence of $H$. pylori than female $(\mathrm{p}<0.01)$. Based on age groups, the highest prevalence was $46.8 \%$ in the $30-39$-year-old group and the lowest prevalence was $40.5 \%$ in the group younger than 30 years. The incidence of $H$. pylori infection decreased with age older than 30 years. The obese (BMI $\geq 25 \mathrm{~kg} / \mathrm{m}^{2}$ ) had a higher rate of HP infection than the non-obese; showed in Table $2(\mathrm{p}<0.01)$.

\section{Associations between lipid profiles and $H$. pylori infection}

Mann-Whitney U test was used to find whether H. pylori infection was relevant to lipid profiles (Tab. 3). The results showed that there were significant associations between triglyceride, HDL cholesterol and $H$. pylori infection $(\mathrm{p}<0.01)$. The positive group had higher levels of triglyceride and lower levels of HDL cholesterol than the negative group. However, the mean values for

Tab. 3. Associations between mean values for lipid profiles and $\mathbf{H}$. pylori infection.

\begin{tabular}{lccc}
\hline & $\begin{array}{c}\text { H. pylori- } \\
\text { positive group }\end{array}$ & $\begin{array}{c}\text { H. pylori- } \\
\text { negative group }\end{array}$ & $\mathrm{p}$ \\
\hline Triglyceride $(\mathrm{mmol} / \mathrm{L})$ & $1.70 \pm 1.43$ & $1.61 \pm 1.32$ & $<0.01$ \\
Cholesterol $(\mathrm{mmol} / \mathrm{L})$ & $5.08 \pm 0.975$ & $.06 \pm 0.98$ & 0.324 \\
HDL cholesterol $(\mathrm{mmol} / \mathrm{L})$ & $1.31 \pm 0.32$ & $1.35 \pm 0.38$ & $<0.01$ \\
LDL cholesterol $(\mathrm{mmol} / \mathrm{L})$ & $2.99 \pm 0.80$ & $2.96 \pm 0.79$ & 0.059 \\
\hline
\end{tabular}

HDL cholesterol - high-density lipoprotein cholesterol; LDL cholesterol - low-density lipoprotein cholesterol. Values are mean \pm standard error of the mean; $p$ values by Mann-Whitney U test. 
total cholesterol and LDL cholesterol in the positive group were higher. Nevertheless, there were no significant differences, when compared with those in the negative group.

\section{Discussion}

Our study showed that the prevalence of $H$. pylori infection was $44.5 \%$ in subjects who took physical examinations at Huashan Hospital Affiliated to Fudan University in Shanghai. The positive rate of $H$. pylori infection was lower than in other cities in China. In Yangzhong, Beijing and Changsha, the prevalence was $63.41 \%$, $46.8 \%$, and $47.2 \%$, respectively $(3,13,14)$. Compared with other countries, the positive rate was also lower. In Korea, the prevalence of the asymptomatic healthy adults was $70.9 \%$ (5). In Gondar and Northern Ireland, it was $65.7 \%$ and $50.5 \%$, respectively $(4,15)$. The reason why the prevalence was lower in Shanghai may lie in the fact that Shanghai was regarded as a well-developed city, while China was considered to be a developing country. There were better conditions relating to hygiene, environmental sanitation and water safety, hence subjects had fewer opportunities to get infected with $H$. pylori. Although the prevalence in Shanghai was lower than in other cities, it was also common among subjects. H. pylori was regarded as Class I carcinogen and it was a pathogen of many diseases such as chronic gastritis, peptic ulcer, gastric malignancies, etc. (1). People who were infected with $H$. pylori usually had not any symptoms in their whole life, so it was necessary to detect whether or not they have infection as early as possible.

Our study found that the males had higher prevalence than females, which was also shown in previous studies $(10,12,16)$. The difference between males and females might be due to different smoking habits, current exposures and physical contacts $(15,17)$. In our study, age was divided into five groups. Among the five age groups, the highest and lowest positive rates were $46.8 \%$ in the age group of 30-39 years and $40.5 \%$ in the age group younger than 30 years. Beyond 30-39 years, the positive rate decreased with increasing age. Previous studies showed that the prevalence of $H$. pylori was higher in the obese which was consistent with our results $(6,7,18)$. The positive rate in the obese group was 47.9 $\%$ which was obviously higher than in the non-obese group $(\mathrm{p}<$ 0.01 ). This might be caused by the fact that in the obese, the innate and adaptive immunity can alter while the immunity deterioration is related to the grade of obesity. They have lower maturation of monocytes into macrophages and reduced polymorphonuclear bactericidal capacity (6). As a result, the obese get easier infected with $H$. pylori than the non-obese.

The associations between triglyceride, HDL cholesterol and $H$. pylori infection were clearly demonstrated in our study, which was showed also in another study (19). The mean value for triglyceride was significantly higher in $H$. pylori-positive group than that in the negative group, while the level of HDL cholesterol was lower in H. pylori infection group. However, significant associations between total cholesterol, LDL cholesterol and H. pylori infection were not confirmed in our study. Although other studies showed there were significant relationships between lipid profiles and $H$. pylori infection, the results were not consistent. Studies showed that H. pylori infection was related to LDL cholesterol but not to other three lipoproteins $(8,9)$. However, another study reported that the infected patients had higher triglyceride, total cholesterol, LDL cholesterol and lower HDL cholesterol (5). Although studies showed contradictory results, there was a general agreement in that $H$. pylori infection had an effect on the lipid profiles. Many studies had reported that triglyceride was independently associated with the risk of cardiovascular disease (20-22). Subjects with elevated level of plasma triglyceride have an increased cardiovascular disease risk. HDL cholesterol was responsible for the removal of free cholesterol from blood vessels to the liver and it also possessed antioxidant and anti-inflammatory properties (23). Decreased level of HDL was associated with an increase in the cardiovascular disease risk. Therefore, $H$. pylori may be a new cardiovascular disease risk factor through increasing the level of triglyceride and decreasing the level of the high-density lipoprotein cholesterol. But the mechanism by which $H$. pylori changed the levels of triglyceride and HDL is still not clear, and therefore needs further research.

In conclusion, this present study demonstrated that the infection of $H$. pylori was common among subjects receiving physical examination in Shanghai. Men and the obese were more likely to be infected with $H$. pylori than women and the non-obese. Individuals at the age of 30-39 years had the highest prevalence compared to other groups. There were significant associations between triglyceride, high-density lipoprotein cholesterol and $H$. pylori infection, indicating that $H$. pylori may be a new cardiovascular risk factor.

\section{References}

1. Brown LM. Helicobacter pylori: epidemiology and routes of transmission. Epidemiol Rev 2000; 22: 283-297.

2. Goodman KJ, Cockburn M. The role of epidemiology in understanding the health effects of Helicobacter pylori. Epidemiology 2001; 12: 266-271.

3. Chen Z, Xu C, Luo L, Xiao J, Yang P, Liu C. Helicobacter pylori infection and gastric mucosa change and blood-lipid in people undergoing the physical examination in Changsha. Zhong nan da xue xue bao Yi xue ban Journal of Central South University Medical sciences 2014; 39: 265-269.

4. Mathewos B, Moges B, Dagnew M. Seroprevalence and trend of Helicobacter pylori infection in Gondar University Hospital among dyspeptic patients, Gondar, North West Ethiopia. BMC Res Notes 2013; 6: 46.

5. Sung KC, Rhee EJ, Ryu SH, Beck SH. Prevalence of Helicobacter pylori infection and its association with cardiovascular risk factors in Korean adults. Internat J Cardiol 2005; 102: 411-417.

6. Arslan E, Atilgan H, Yavasoglu I. The prevalence of Helicobacter pylori in obese subjects. Eur J Intern Med 2009; 20: 695-697.

7. Xu C, Yan M, Sun Y et al. Prevalence of Helicobacter pylori infection and its relation with body mass index in a Chinese population. Helicobacter 2014; 19: 437-442.

8. Kim HL, Jeon HH, Park IY, Choi JM, Kang JS, Min KW. Helicobacter pylori infection is associated with elevated low density lipoprotein cholesterol levels in elderly Koreans. J Kor Med Sci 2011; 26: 654-658.

9. Shiotani A, Miyanishi T, Uedo N, Iishi H. Helicobacter pylori infection is associated with reduced circulating ghrelin levels independent of body mass index. Helicobacter 2005; 10: 373-378. 


\section{$521-524$}

10. Satoh H, Saijo Y, Yoshioka E, Tsutsui H. Helicobacter Pylori Infection is a Significant Risk for Modified Lipid Profile in Japanese Male Subjects. J Atheroscler Thromb 2010; 17: 1041-1048.

11. Takashima T, Adachi K, Kawamura A et al. Cardiovascular risk factors in subjects with Helicobacter pylori infection. Helicobacter 2002; 7: 86-90.

12. Longo-Mbenza B, Nsenga JN, Mokondjimobe $E$ et al. Helicobacter pylori infection is identified as a cardiovascular risk factor in Central Africans. Vasc Health Risk Manag 2012; 6: 455-461.

13. Cheng H, Hu F, Zhang $L$ et al. Prevalence of Helicobacter pylori infection and identification of risk factors in rural and urban Beijing, China. Helicobacter 2009; 14: 128-133.

14. Zhu Y, Zhou X, Wu J, Su J, Zhang G. Risk Factors and Prevalence of Helicobacter pylori Infection in Persistent High Incidence Area of Gastric Carcinoma in Yangzhong City. Gastroenterol Res Pract 2014; 2014: 481365 .

15. Murray LJ, McCrum EE, Evans AE, Bamford KB. Epidemiology of Helicobacter pylori infection among 4742 randomly selected subjects from Northern Ireland. Int J Epidemiol 1997; 26: 880-887.

16. Chong VH, Lim KC, Rajendran N. Prevalence of active Helicobacter pylori infection among patients referred for endoscopy in Brunei Darussalam. Singap Med J 2008; 49: 42-46.
17. Replogle ML, Glaser SL, Hiatt RA, Parsonnet J. Biologic Sex as a Risk Factor for Helicobacter-Pylori Infection in Healthy-Young Adults. Am J Epidemiol 1995; 142: 856-863.

18. Yang GH, Wu JS, Yang YC, Huang YH, Lu FH, Chang CJ. Obesity associated with increased risk of gastric Helicobacter pylori infection in an elderly Chinese population. J Amer Geriat Soc 2014; 62:1 90-192.

19. Niemela S, Karttunen T, Korhonen T et al. Could Helicobacter pylori infection increase the risk of coronary heart disease by modifying serum lipid concentrations? Heart 1996; 75: 573-575.

20. Boullart AC, de Graaf J, Stalenhoef AF. Serum triglycerides and risk of cardiovascular disease. Biochim Biophys Acta 2012; 1821: 867-875.

21. Patel A, Barzi F, Jamrozik $\mathbf{K}$ et al. Serum triglycerides as a risk factor for cardiovascular diseases in the Asia-Pacific region. Circulation 2004; 110: 2678-2686.

22. Sarwar N, Danesh J, Eiriksdottir G et al. Triglycerides and the risk of coronary heart disease: 10,158 incident cases among 262,525 participants in 29 Western prospective studies. Circulation 2007; 115: 450-458.

23. Sviridov D, Mukhamedova N, Remaley AT, Chin-Dusting J, Nestel P. Antiatherogenic functionality of high density lipoprotein: how much versus how good. J Atheroscler Thromb 2008; 15: 52-62.

Received March 9, 2016. Accepted March 19, 2016. 